\title{
Journey of Women: Puberty to Post-menopause
}

\author{
Apurva Shrivastava and Deepika MLN* \\ Department of Clinical Genetics, Institute of Genetics and Hospital for Genetic Diseases, Osmania University, Hyderabad, Telangana, India
}

"Corresponding author: Deepika MLN, Department of Clinical Genetics, Institute of Genetics \& Hospital for Genetic Diseases, Osmania University, Hyderabad, Telangana, India, Tel: +91-9885617014; Fax: +91-040- 23403681; E-mail: mlndeepika@gmail.com

Received: 09 Nov, 2017 | Accepted: 30 Jan, 2018 | Published: 06 Feb, 2018

Citation: Shrivastava A, Deepika MLN (2018) Journey of Women: Puberty to Post-menopause. Gynecol Women's Health Res 1(1): dx.doi.org/10.16966/2689-3096.107

Copyright: (c) 2018 Deepika MLN, et al. This is an open-access article distributed under the terms of the Creative Commons Attribution License, which permits unrestricted use, distribution, and reproduction in any medium, provided the original author and source are credited.

\section{Abstract}

Women are facing several health hazards that have an adverse effect on them and their offspring. In this article, the potential health risk factors and repercussions in women are evaluated so that sustenance of well-being is maintained and appropriate strategic interventions are made at critical stages such as at childhood, adolescence and pregnancy. Weakened and restricted access to public health system for low socio-economic status women and unregulated growth of the private sector in healthcare is leading to a near-total eclipse of availability and accessibility of universal and comprehensive healthcare.

Keywords: Journey; Adolescence; Adulthood; Pre-menopause; Post-menopause

\section{Introduction}

The journey of womanhood is wonderful yet complex. Right from being a child to a teenager and later a woman, the female body undergoes innumerable changes. However beautiful and significant these changes might be, there are certain underlying causes that affect one's physical and mental health. More often the changes are accepted rather than making efforts to understand and adjusting the body towards the change. Since most females are married during adolescence [1], they do not understand their needs and restrictions properly. Any effect on the genotypic and phenotypic aspects of health on a woman at any stage in life can lead to serious health complications. Often, the lack of understanding and knowledge of these complications by a girl leads to serious health issues, and so, in this article we intend to enlighten everyone with certain diseases, psychological problems and general concerns that are associated with the different stages in the development of the female body $[2,3]$.

\section{Adolescence}

The major landmark of girl's puberty is menarche, which occurs on an average between ages 10-16 [4]. Analogous to but distinct from changes experienced by boys, a girl's body undergoes gradual changes during puberty. Puberty is the process of physical changes by which a child's body matures into an adult body capable of sexual reproduction to enable fertilisation. Signals from the brain to the gonads (ovaries or testes) for the release of hormones, such as the Gonadotropinreleasing hormone $(\mathrm{GnRH})$ that precede Luteinising hormone $(\mathrm{LH})$ and Follicle-stimulating hormone (FSH), initiates the physical changes. These hormones stimulate effective changes on several functions associated with sexual development such as transformative growth of bones, muscle, skin, hair and sexual organs, with height and weight being the visible factors that change rapidly at the onset of puberty, and later, become slow in the adult body. There are various problems that can occur at puberty. Serious health complications arise when there are many deviations from a normal development. These deviations can arise due to environmental or genetic factors and include issues such as hormonal imbalance, skin outbreaks, no development of sexual characters, complete absence or delay in initiation of menses / amenorrhea etc. [5].

Early onset of menarche and increased prevalence of obesity-related menstrual disorders are attributed to an increase in the rate of childhood obesity around the world. Exposure to environmental toxins, namely hormonally active endocrine disruptors result in increased rates of menstrual and reproductive disorders in endemic areas. Thyroid hormones are one class of hormones that are essential for normal growth, sexual development and reproductive function. During puberty, changes in thyroid functions and an increase in thyroid volume occur as an adaptation to body and sexual development. A short period of overactive thyroid (hyperthyroidism) seems not to have major negative effects [6]. But people with underactive thyroid (Hypothyroidism-Hashimoto's hypothyroidism) might feel depressed and sluggish, might gain weight, even 
with the right diet and exercise. Adolescents suffer since this affects negatively on sexual development, creates difficulty in concentrating and leads to skin and hair problems. Diagnosis is easily done with the help of blood tests and treatment usually involves measures to restore thyroid levels, known as thyroid replacement therapy. Long term stress may cause many deleterious consequences leading to various endocrine disorders of the thyroid gland, anxiety, psychological disorders, sexual dysfunction and several hormone-related disorders [7].

Müllerian agenesis, the absence of uterus, is one such disorder and is the aetiology in $15 \%$ of cases of primary amenorrhea [8]. In Müllerian agenesis, while the female (46 $\mathrm{XX}$ ) enters menarche, develops secondary sexual characters at the right age, the vagina can eventually shorten and ultimately close, leading to painful intercourse. This leads to complete absence or partial absence of cervix, uterus and vagina. No proper treatment has been found for Müllerian agenesis, but pregnancy can be attained by IVF techniques and surrogacy. Primary amenorrhea is a condition when menstruation is not initiated even at the age of 16 and no secondary sexual characters such as breast development have been observed by the age of 13 . This usually occurs because of genetic or anatomic abnormalities. Females with abnormal sex chromosomes (XOhypogonadotrophic hypogonadism-Turner's syndrome or XY) are the ones to be affected. Secondary amenorrhea results in withdrawal in periods after a certain period of menses. The symptoms are the duration of cycle being longer than 35 days. Premature ovarian failure due to abnormal karyotype is the genetic causes of secondary amenorrhea [9].

It is often not understood by the girl as to why such changes are happening and therefore, proper education regarding the changes and management must be provided for easy shift over and adjustment to the change. A girl is under pressure to be accepted within the norms while not being capable of accepting the changes in her body and state of mind. At this age, proper guidance can only be provided by the mother or a close relative as it becomes very important to understand oneself at this age [10].

\section{Early adulthood}

Today women have a lot on their minds. Apart from personal understanding of health and diseases, the main issue is women are hesitant about revealing their problems. At the reproductive age, a woman should be able to openly discuss any health problems with her family and doctor. It is usually difficult to reveal problems to clinicians which makes openness towards healthcare services more important. Also, there is strong correlation between illiteracy and women's health [11]. Women in rural and most of the urban parts of India do not clearly understand the concept of contraception due to it being a taboo to share information about, because of which they are prone to attain a sexually transmitted disease $[12,13]$.

Current generation's pressing issue is infertility. Stress, bad food habits (fat foods cooked in oils with high levels of trans-fat) leading to obesity, too much or too less exercise, constant exposure to indoor pollutant, chemicals at work place, industrial pollutant, cigarette smoke, radiations and pollution affects a person's reproductive system, be it a man or woman [14]. The pollutants are particularly dangerous, and for women on coming in contact with them, either through breathing or skin contact, can lead to long-term damage. Infertility being asymptomatic in nature, one can keep a tab on their menstrual cycle. A cycle too long (35 days) or too short (21 days), absent or irregular must be checked with a doctor, especially if one is between 30-40 years of age. There are many causes for infertility such as damaged fallopian tubes, hormonal (overactive or underactive thyroid gland), uterine, cervical and ovulation disorders (premature ovarian failure and PCOS) [15].

PCOS is a major problem in most women who are likely to conceive. The genes for PCOS can be inherited from either the mother or father, with family history of PCOS or women with irregular periods is known [16-18]. Cysts in the ovaries can lead to hormonal imbalance i.e., release of more male hormones such as androgens, causing acne and growth of facial hair along with arrest of ovulation. Though the cysts are not harmful, they may create irregular periods and problems during pregnancy and if not treated, may cause complex diseases. There is no particular cure for PCOS but, hormone capsules can be taken to balance their levels and a healthy lifestyle can help avoid infertility and miscarriages. Fortunately, science of treating infertility has progressed and treatments can be done by laparoscopy, artificial insemination, in vitro fertilization, etc. [19].

During pregnancy, it becomes crucial that the body receives sufficient amounts of vitamins, iron, calcium and minerals. For proper guidance in this subject, on which most of the women are not well-informed, being open to the doctor and asking any doubts and questions will help in better progression of this phase. Such a facility is difficult to obtain in rural environment. Good amount of energy, nutritional intakes, exercises and proper breathing are necessary to avoid miscarriages. Any pregnancy can be classified into low risk or high risk pregnancy and healthcare services are required for both, but, there are not many services that help women to manage a high risk pregnancy. Various genetic links and biological markers have been identified to be associated with pregnancy. Thyroid auto-antibodies serve as markers for at-risk pregnancies [20]. Presence of thyroid auto antibodies reflects a generalized activation of the immune system and a generally heightened autoimmune reactivity against the feto-placental unit. Women with thyroid auto antibodies could have a more generalized immune imbalance. Higher the amount of thyroid in the blood, higher is the risk of pregnancy loss [21]. There are a few studies showing that thyroxine treatment may be effective in reducing the number of miscarriages when given during the early stages of pregnancy [22].

Miscarriages are caused due to external as well as internal problems. Lupus anticoagulant [23] was among the first 
autoimmune disorders to be recorded as a reason for recurrent abortions [24,25]. Among other autoimmune disorders is the Antiphospholipid syndrome, which is defined by the presence of at least one clinical manifestation (venous/arterial thrombosis or obstetric complications) and antiphospholipid antibodies, that recognise negatively charged plasma proteins, and include anti-cardiolipin, anti- $\beta$ - 2 glycoprotein and lupus anticoagulant antibodies, etc. [24]. Aneuploidy may also be a cause of a random spontaneous as well as recurrent pregnancy loss and is more common with advanced reproductive age reflecting decreased germ cell quality. Thrombophilia (factor V Leiden and prothrombin G20210A mutation), decreased maternal immune tolerance towards the foetus, paternal sharing of HLA genes, reduced ovarian reserve, being in a stressful atmosphere can all lead to repeated abortions [26]. Recurrent miscarriage in itself is associated with development of coronary artery disease and an increased risk of ovarian cancers at later stages [27].

Women with a history of recurrent miscarriage are at risk of developing preeclampsia in subsequent pregnancy [28]. A high blood pressure and high amount of protein in urine during pregnancy are the symptoms of preeclampsia. Chronic hypertension, prior history of preeclampsia, family history of preeclampsia, advanced maternal age ( $>35$ years), obesity, antiphospholipid antibody syndrome, multiple gestation, having donated a kidney, sub-clinical hypothyroidism or thyroid antibodies are all the factors that can cause preeclampsia [29]. Also, the presence of anti-thyroid antibodies may react against the structures of the placenta or fertilized egg and cause problems in embryo implantation [30,31].

Pregnancy induced hypertension is different from preeclampsia because there are no proteins in urine. Having a blood pressure is higher than 140/90 measured on two separate occasions, more than 6 hours apart, without the presence of protein in the urine and diagnosed after 20 weeks of gestation is a symptom of gestational hypertension [32].

Women with bad obstetric history $(\mathrm{BOH})$ present antiphospholipid antibodies, hypertension, malpresentation, cervical incompetence, preterm deliveries and caesarean section. Mothers with $\mathrm{BOH}$ are four times likely to deliver a low birth weight baby [33]. Age, obesity and high parity have been shown to be independent risk factors for RPL and stillbirth [34].

Maternal mortality is the most common form of death in developing countries [35,36]. In addition, numerous women in most parts of India have closely spaced births that also increase the health risk for the mothers. Death due to pregnancy problems are mostly because the underlying issue often goes unnoticed [37].

\section{Late adulthood-pre menopause}

Gaining weight, breasts become sore and lumpy, loss of sex drive, changed skin texture, mood swings and irregular periods are few signs of pre menopause and are caused by hormone imbalances (excess of the hormone estrogen and deficiency of the hormone progesterone), prolonged physical or emotional stress, carbohydrate rich food and frequent exposure to pollutants. Menstrual cycles may lengthen or shorten or even not release an egg (ovulate). Menopause-like symptoms, such as hot flashes, sleep problems and vaginal dryness may also occur. Most of the women in pre-menopause have decreased bone density leading to brittle bones, vulnerable to fractures. Female body specific health issues are topped by breast cancer, cervical cancer and ovarian cancer. Symptoms for such diseases are observable and one must know what signs are to be looked out for. For breast cancer, swelling up of one breast or presence of lumps in breasts; for cervical cancer it can be spotting of blood irregularly and pain in abdomen are a few red flags [38,39].

More often than not, women tend to disregard such symptoms as temporary or related to periods. Also, women pose a greater risk for developing diabetes, hypertension and thyroid problems in this decade. At this stage is becomes imperative to visit a gynaecologist regularly and not forego any symptoms $[40,41]$.

\section{Menopause}

This stage is majorly characterised by complete cessation of the predictable menstrual cyclicity of the woman that progresses with aging. There is a steady decline in the number of ovarian follicles, the amount of ovarian hormones and fertility. As there is a decrease in endocrine function of the ovaries, a condition known as ovarian insufficiency arises. The mean age of menopause occurring is 51 years [42-44] and depends on difference of geography, race/ethnicity, socio-economic status, menstrual and reproductive history, body mass index and genetics. The classic symptoms are hot flashes and night sweats, menstrual disorders, muscular and joint pains, sleep disorders [45,46], psychological changes and sweating [47].

Menopause is a highly debated subject when it comes to relating it with chronic diseases like cancers, osteoporosis and heart conditions [48]. Decreased libido, genital inflammation and painful sexual intercourse, usually leading to avoidance of sex completely can be seen in approximately $60 \%$ of patients [49]. Women who suffer with depression earlier have a five-fold higher risk of developing depression during the pre- and perimenopausal stage itself. Not only this, factors such as living in urban areas, low socio-economic status and illiteracy also add to the development of depression. Muscle and joint pain worsen during menopause since there is a decrease in bone mineral density [50]. Severe bone loss is associated with the decrease in synthesis of estrogen, which is an important in pathogenesis of osteoporosis. Hypoestrogenism helps in the destructive action of osteoclasts and inhibitory action of osteoblasts, which all lead to osteoporosis [51].

In many such ways, being at menopause causes a lot of stress on mind and body. But irrespective of race or sociodemographic factors, every woman suffers from these ailments and hence, attention must be drawn towards improving 
medical healthcare and making professional assistance available. Hormone replacement therapy (HRT) and Estrogen Replacement therapy has large benefits in prevention of heart and bone diseases and is commonly undergone by women to cope up with the changing conditions. Anti-depressants at low doses could be administered to manage the hot-flashes, which is helpful in a few cases since some women are not able to take estrogen due to allergic reactions or other health conditions. Being open about the frequency and intensity of hot-flashes with the doctor can allow for prescription of the right kind of medicine that you can take. Treatment for osteoporosis with the help of medications to strengthen your bones can also be followed [52].

\section{Post-menopause}

In post-menopausal stage, the most common reason for women not being able to give much importance to their physical state is the psychological state. Due to the advancement of menopause, a woman tends towards depression and undergoes various psychological problems. These lead to serious problems such as insomnia or sleep apnea, where a person stops breathing repeatedly during the night causing awakenings, a condition which is linked to increase in risk of heart attacks and strokes. There is also a severe decline in estrogen production at this stage, which is a major risk factor for heart diseases caused in women, cerebral ischemia and neurodegenerative disorders like dementia and Alzheimer's [53]. Other issues that occur are presbycusis (loss of hearing), osteoporosis and osteoarthritis [52].

Hypertension, diabetes, breast cancer and cervical cancer are the most common diseases faced by women in age group. Diabetes, a common problem of this age group, must be checked for in every three months, in case the person is nondiabetic. A diabetic patient must take care of their diet, have proper amount of exercise, and most importantly be aware of the long term complication such as diabetic nephropathy and retinopathy, gangrene, heart problems, etc. Any injury must be taken care of immediately as it takes longer than usual to heal in case of diabetes [54,55].

Ageing results in deficiency of calcium and loosening of muscles for which a woman must focus on physical activity which can be gained by regular exercises. Also a balanced diet having the vital minerals and vitamins is of utmost importance as body requirements keep changing to cope with the changes. A good amount of sleep of at least 6-8 hours is important so as to not disturb the biological rhythm. Maintenance of hygiene and having a proper diet which includes rich source of vitamins, proteins and essential minerals is a key to coping up with body's changes [56].

\section{Conclusion}

A woman's body is always on duty and she must take care of what is happening intrinsic and extrinsic. No matter how far science has advanced, prevention is always better than cure. And as it's said, a healthy mind, a healthy body can allow a woman to make her mark in the world. Clinical assessment along with a healthy lifestyle and routine check-ups are crucial at every stage of women's development.

\section{References}

1. UNICEF (2014) Ending Child Marriage: Progress and prospects, Page 6, New York.

2. Trickett PK, Noll JG, Putnam FW (2011) The impact of sexual abuse on female development: Lessons from a multigenerational, longitudinal research study. Dev Psychopathol 23: 453-476.

3. Graberc, JA, Brooks-Gunn J, Warren MP (1995) The Antecedents of Menarcheal Age: Heredity, Family Environment, and Stressful Life Events. Child Dev 66: 346-359.

4. Rees M (1995) The age of menarche. ORGYN 4: 2-4.

5. Shayesteh Jahanfar (2012) Genetic and environmental determinants of menstrual characteristics. Indian J Hum Genet 18: 187-192.

6. Weber G, Vigone MC, Stroppa L, Chiumello G (2003) Thyroid function and puberty. J Pediatr Endocrinol Metab 16: 253-257.

7. Salam Ranabir and Reetu K (2011) Stress and hormones. Indian J Endocrinol Metab15: 18-22.

8. Deligeoroglou E, Athanasopoulos N, Tsimaris P, Dimopoulos KD, Vrachnis N, et al. (2010) Evaluation and management of adolescent amenorrhea. Ann N Y Acad Sci 1205-1223.

9. Jiao X, Qin C, Li J, Qin Y, Gao X, et al. (2012) Cytogenetic analysis of 531 Chinese women with premature ovarian failure. Hum Reprod 27: 2201-2207.

10. Galambos NL, Barker ET, Almeida DM (2003) Parents Do Matter: Trajectories of Change in Externalizing and Internalizing Problems in Early Adolescence. Child Dev 74: 578-594.

11. Puri S, Bhatia V, Swami HM, Singh A, Sehgal A, et al. (2007) Awareness of emergency contraception among female college students in Chandigarh, India. Indian J Med Sci 61: 338-346.

12. Gupta N, Mathur AK, Singh MP, Saxena NC (2004) Reproductive health awareness of school-going, unmarried, rural adolescents. Indian J Pediatr 71: 797-801.

13. León FR, Lundgren $R$, Sinai I, Sinha R, Jennings $V$ (2014) Increasing literate and illiterate women's met need for contraception via empowerment: a quasi-experiment in rural India. Reprod Health11: 74.

14. Bano S, Wani KA (2012) The Health Conditions of Rural Localities of Madhya Pradesh. Eur J Biol Sci 4: 20-25.

15. R Blundell (2007) Causes of infertility. Int J Med Adv Sci3: 63-65.

16. Kahsar-Miller MD, Nixon C, Boots LR, Go RC, Azziz R (2001) Prevalence of polycystic ovary syndrome (PCOS) in first-degree relatives of patients with PCOS. Fertil Steril 75: 53-58.

17. Sam S, Legro RS, Essah PA, Apridonidze T, Dunaif A (2006) Evidence for metabolic and reproductive phenotypes in mothers of women with polycystic ovary syndrome. Proc Natl Acad Sci U S A 103: 7030-7035.

18. Legro RS, Driscoll D, Strauss JF, Fox J, Dunaif A (1998) Evidence for a genetic basis for hyperandrogenemia in polycystic ovary syndrome. Proc Natl Acad Sci U S A 95: 14956-14960. 
19. Yoo Shin Kim and Tae Ki Yoon (2007) Treatment of Female Infertility. J Korean Med Assoc 50: 406-414.

20. Francis S Balucan, Syed A Morshed, Terry F Davies (2013) Thyroid Autoantibodies in Pregnancy: Their Role, Regulation and Clinical Relevance. J Thyroid Res 2013: 182472.

21. Clinical Thyroidology for the Public (2014) American Thyroid Association 7.

22. Kennedy RL, Malabu UH, Jarrod G, Nigam P, Kannan K, et al. (2010) Thyroid function and pregnancy: Before, during and beyond. J Obstet Gynaecol 30: 774-783.

23. Lubbe WF, Butler WS, Palmer SJ, Liggins GC (1984) Lupus anticoagulant in pregnancy. Br J Obstet Gynaecol 91: 357-363.

24. Perricone C, Carolis C, Perricone R (2012) Pregnancy and autoimmunity: a common problem. Best Pract Res Clin Rheumatol 26: 47-60.

25. Andreoli L, Fredi M, Nalli C, Reggia R, Lojacono A, Motta M, et al. (2012)Pregnancy implications for systemic lupus erythematosus and the antiphospholipid syndrome. J Autoimmun 38: 197-208.

26. Qu F, Wu Y, Zhu YH, Barry J, Ding T, et al. (2017) The association between psychological stress and miscarriage: A systematic review and meta-analysis. Sci Rep 7: 1731.

27. Oliver-Williams CT, Heydon EE, Smith GC, Wood AM (2013) Miscarriage and Future Maternal Cardiovascular Disease: A Systematic Review and Meta-Analysis. Heart 99: 1636-1644.

28. Trogstad Lill, Magnus Per, Moffett Ashley, Stoltenberg Camilla (2009) The effect of recurrent miscarriage and infertility on the risk of pre-clampsia. BJOG 116: 108-113.

29. Garg AX, Immaculate FN, Eric M, Jessica MS, John JK, et al. (2014) Gestational Hypertension and Preeclampsia in Living Kidney Donors. N Engl J Med 372: 124-133.

30. Negro R, Formoso G, Mangieri T, Pezzarossa A, Dazzi D, et al. (2006) Levothyroxine treatment in euthyroid pregnant women with autoimmune thyroid disease: effects on obstetrical complications. The J Clin Endocrinol Metab 91: 2587-2591.

31. Miyakis S, Lockshin MD, Atsumi T, Branch DW, Brey RL, et al. (2006) International consensus statement on an update of the classification criteria for definite antiphospholipid syndrome (APS). J Thromb Haemost 4: 295-306.

32. AlessiaMammaro, Sabina Carrara, Alessandro Cavaliere, Santina Ermito, AngelaDinatale, et al. (2009) Hypertensive disorders of pregnancy. J Prenat Med 3: 1-5.

33. Champa Koppad, Lakshmi KS (2014) Immunological Causes of Bad Obstetric History. J Evi Med Healthcare 1: 2086-2099.

34. G Singh and K Sidhu (2010) Bad Obstetric History: A Prospective Study. Med J Armed Forces India 66: 117-120.

35. Conde-Agudelo A, Belizan JM, Lammers C (2004) Maternalperinatal morbidity and mortality associated with adolescent pregnancy in Latin America: Cross-sectional study. Am J Obstet Gynecol 192: 342-349.

36. Filippi V, Chou D, Ronsmans C, Graham W, Say L, et al. (2016) Levels and Causes of Maternal Mortality and Morbidity. 3rd Edition (Volume 2), The International Bank for Reconstruction and Development / The World Bank, Washington (DC), USA.

37. Zhang B-N, Cao X-C, Chen J-Y, Chen Jie, Fu Li, et al. (2012) Guidelines on the diagnosis and treatment of breast cancer (2011 edition). Gland Surg 1: 39-61.
38. Geneva: World Health Organization (2014) Comprehensive Cervical Cancer Control, 2nd edition. A Guide to Essential Practice.

39. Marcia Frellick (2016) Early or Late Menopause Ups Risk for Type 2 Diabetes. Medscape.

40. Gierach GL1, Johnson BD, Bairey Merz CN, Kelsey SF, Bittner V, et al. (2006) Hypertension, Menopause, and Coronary Artery Disease Risk in the Women's Ischemia Syndrome Evaluation (WISE) Study. J Am Coll Cardiol 47: S50-S58.

41. Patton GC, Coffey C, Sawyer SM, Viner RM, Haller DM, Bose K, et al. (2009) Global patterns of mortality in young people: a systematic analysis of population health data. Lancet 374: 881-892.

42. McKinlay SM, Brambilla DJ, Posner JG (1992) The normal menopause transition. Maturitas 14: 103-115.

43. Greendale G, Hogan P, Kritz-Silverstein D, Donna, Langer, et al. (1995) Age at menopause in women participating in the postmenopausal estrogen/progestins interventions (PEPI) trial: an example of bias introduced by selection criteria. Menopause 2: 27-34.

44. Luoto R, Laprio J, Uutela A (1994) Age at natural menopause and sociodemographic status in Finland. Am J Epidemiol 139: 64-76.

45. Joffe H, Massler A, Sharkey KM (2010) Evaluation and Management of Sleep Disturbance During the Menopause Transition. Semin Reprod Med 28: 404-421.

46. SimaKiani rad, SedigheForouhari, Dehaghani AS, Vafaei $H$, Sayadi $M$, et al. (2016)The effect of salvia officinalis tablet on hot flashes, night sweating, and estradiol hormone in postmenopausal women. Int J Med Res Health Sci 5: 257-263.

47. Dalal PK, Agarwal M (2015) Postmenopausal syndrome. Indian J Psychiatry 57: S222-S232.

48. Kravitz HM, Avery E, Sowers M, Bromberger JT, Owens JF, et al. (2011) Relationships between menopausal and mood symptoms and eeg sleep measures in a multi-ethnic sample of middle-aged women: the SWAN sleep study. Sleep 34: 1221-1232.

49. Bitzer J, Brandenburg U (2009) Psychotherapeutic interventions for female sexual dysfunction. Maturitas 63: 160-163.

50. Management of osteoporosis in postmenopausal women: 2010 position statement of The North American Menopause Society (2010) Menopause 17: 25-54.

51. Okman-Kilic T (2015) Estrogen deficiency and osteoporosis. In: Dionyssiotis Y(eds) Advances in Osteoporosis, InTech.

52. Osteoporosis and Arthritis: Two Common but Different Conditions (2016) NIH Osteoporosis and related Bone Diseases National Resource Center 16-7893.

53. Dye RV, Miller KJ, Singer EJ, Levine AJ (2012) Hormone Replacement Therapy and Risk for Neurodegenerative Diseases. Int J Alzheimers Dis 2012: 258454.

54. Snyder RJ (2005) Treatment of nonhealing ulcers with allografts. Clin Dermatol 23: 388-395.

55. Moreo K (2005) Understanding and overcoming the challenges of effective case management for patients with chronic wounds. Case Manager 16: 62-67.

56. Chernoff R (2005) Micronutrient requirements in older women. Am J Clin Nutr 81: 1240S-1245S. 